STRUCTURAL SCIENCE CRYSTAL ENGINEERING MATERIALS

ISSN 2052-5206

\section{Absolute structure determination: pushing the limits}

\author{
A. L. Spek*
}

Utrecht University, Bijvoet Center for Biomolecular Research, Padualaan 8, 3584 CH Utrecht, The Netherlands. *Correspondence e-mail: a.l.spek@uu.nl

It was the Softenon disaster (see Wikipedia) that made the pharmaceutical industry fully aware of the importance of knowing the enantiomeric purity and chirality of drugs and their metabolites. This disaster involved the chiral drug Thalidomide (Fig. 1) that was sold in the 1950s as a racemate under various brand names such as Contergan and Softenon. It was shown in the early 1960s that only the $R$-enantiomer has the intended pharmaceutical effect and that the $S$-enantiomer, when the drug is used by pregnant females, may lead to serious miscarriages.

Until the 1950s, the chirality of a compound could only be determined by chemical methods relative to the arbitrarily chosen 'absolute configuration' of (+)-R-glyceraldehyde (the Fischer-Rosanoff Convention). For a long time it was thought that X-rays could not be used to distinguish between enantiomeric structures (Friedel's law). It was J. M. Bijvoet's organic chemistry colleague, F. Kögl, working on the isolation of natural products and on a chirality-related cancer theory, who inspired Bijvoet to reinvestigate the possibility to directly determine the chirality of molecules such as natural and unnatural amino acids with X-ray diffraction techniques without reference to glyceraldehyde. Inspiration for the latter was gleaned from the largely forgotten paper by Coster et al. (1930) on the association of the two macroscopically distinguishable crystal 111 faces with the microscopic $\mathrm{Zn}$ and $\mathrm{S}$ layers in crystals of the inorganic compound zinc blende, ZnS, using X-ray techniques. Bijvoet realised that Friedel's law did not apply when resonant scattering (anomalous dispersion) was taken into account. Not only the stacking polarity in crystals but also the chirality of molecules could be determined using $\mathrm{X}$-ray techniques. The current notion of 'absolute structure' covers both polarity and chirality determination.

The first absolute structure determination of an organic compound, as proof of the principle, was carried out for sodium rubidium (+)-tartrate in 1950 (Bijvoet et al., 1951). This assignment was based on careful measurement of the difference in intensity of Friedel pairs (i.e. $h k l$ and $\bar{h} \bar{k} \bar{l}$ ) of reflections. This was a significant experimental feat at that time in view of the long exposure time required for taking the Weissenberg diffraction images (over $200 \mathrm{~h}$ ), hampered by unstable X-ray sources. The sign of the difference in intensity of a small number of Friedel pairs showing a large observed intensity difference was compared with the associated sign of the intensity difference calculated from the structure model. A $\mathrm{Zr} K \alpha$ X-ray source along with $\mathrm{Rb}^{+}$as the heavy atom was chosen for a maximum anomalous difference signal. By pure chance, the determined absolute structure turned out to be consistent with the arbitrary choice made by Fischer \& Rosanoff, which was good for science (avoiding confusion) but obviously with less impact in showing the power of the X-ray technique than when it would have been otherwise. Half a century later the absolute structure assignment to sodium rubidium (+)-tartrate was reaffirmed using state-of-the-art techniques (Lutz \& Schreurs, 2008).

With the advancement of the diffraction and computer hardware and the inclusion of anomalous dispersion contributions into the structure refinement software, it became customary to refine both enantiomeric models of a determined structure and keep the one with the lowest $R$-value as that representing the true absolute structure. Probability tests (e.g. the Hamilton test) to determine the validity of the chosen absolute structure were often problematic; neither were the possibilities of an enantiomerically impure sample or of a racemic mixture addressed. This situation was finally resolved with the introduction of the Flack parameter (Flack, 1983) that is based on the physically meaningful inversion twinning model defined in the range $0.0-1.0$, where the limiting 


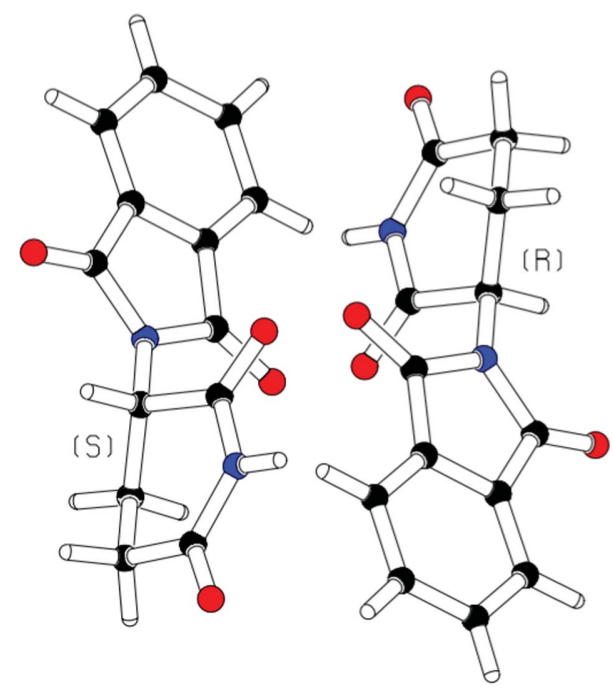

Figure 1

The drug $(R / S)$ Thalidomide.

values indicate the two pure enantiomers and an intermediate value a racemic mixture. As a refined parameter, the Flack parameter value comes with an associated s.u. value that can be used to determine the statistical reliability of the assignment of absolute structure to an enantiomerically pure compound.

Over time, experience was gained with the Flack parameter values determined for enantiomerically pure compounds in relation to their associated s.u.s. Absolute structure assignment turned out to be generally statistically reliable for compounds containing heavy atoms with significant resonant power. Unfortunately, this was rarely the case for light atom structures containing only atoms of types $\mathrm{O}, \mathrm{N}, \mathrm{C}$ and $\mathrm{H}$ measured with Mo $K \alpha$ radiation. Interestingly, in cases where the absolute structure of enantiomerically pure compounds was known, Flack parameter values resulted in values close to zero but with s.u. values too large for statistical reliability, suggesting that those s.u. values might be overestimated.

Absolute structure determination of light atom structures is of great interest in pharmaceutical research. To address the reliability issue as measured by the s.u., a new approach based on Bayesian statistics was taken (Hooft et al., 2008). Using the same inversion twinning model, a Flack parameter look-alike parameter (the Hooft $y$ parameter) could be determined with a generally lower s.u. by a factor of 2 or 3. Subsequently, similar approaches (collectively addressed as post-refinement methods) have been implemented in various software packages (e.g. the Parsons parameter in SHELXL; Sheldrick, 2015). The Parsons quotient approach and the associated Parsons parameter are discussed in Parsons et al. (2013) along with a comparison of the outcome of the post-refinement Parsons and Hooft parameter determinations with the value of the Flack parameter as determined with the inversion twin refinement approach for a set of 23 light atom structures. Parsons and Hooft parameter calculations are now part of the IUCr PLATON/checkCIF procedures (Spek, 2003).

The paper 'Why Direct and Post-refinement Determinations of Absolute Structure May Give Different Results' by Watkin \& Cooper (2016) in this issue describes the currently available techniques for absolute structure determination and investigates the question raised in the title of the paper. Extensive tests were done on 28 materials using the six different procedures as implemented in the CRYSTALS (Betteridge et al., 2003) package to estimate the value of the Flack parameter and its s.u.

One of the differences between the refined and the postrefinement approaches to determine the absolute structure is that the former may work fine with a data set without Friedel (or Bijvoet) pairs where the latter requires an essentially complete set of Friedel pairs. The post-refinement procedures allow for various weighting schemes to be applied to the Friedel pairs, including the removal of obvious outliers. The general advice given by Watkin \& Cooper (2016) in their paper is to use both approaches to see whether their outcome is generally consistent and, if not, to investigate the reason.

\section{References}

Betteridge, P. W., Carruthers, J. R., Cooper, R. I., Prout, K. \& Watkin, D. J. (2003). J. Appl. Cryst. 36, 1487.

Bijvoet, J. M., Peerdeman, A. F. \& van Bommel, A. J. (1951). Nature, 168, 171-172.

Coster, D., Knol, K. S. \& Prins, J. A. (1930). Z. Phys. 63, 345-369.

Flack, H. D. (1983). Acta Cryst. A39, 876-881.

Hooft, R. W. W., Straver, L. H. \& Spek, A. L. (2008). J. Appl. Cryst. 41, 96-103.

Lutz, M. \& Schreurs, A. M. M. (2008). Acta Cryst. C64, m296-m299.

Parsons, S., Flack, H. D. \& Wagner, T. (2013). Acta Cryst. B69, 249 259.

Sheldrick, G. M. (2015). Acta Cryst. A71, 3-8.

Spek, A. L. (2003). J. Appl. Cryst. 36, 7-13.

Watkin, D. J. \& Cooper, R. I. (2016). Acta Cryst. B72, 661-683. 